Geometry $8 \mathcal{T}$ Topology

Volume 5 (2001) 579-608

Published: 18 June 2001

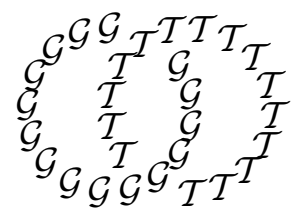

\title{
Lefschetz pencils and divisors in moduli space
}

\author{
IVAN SMITH \\ New College, Oxford \\ OX1 3BN, UK \\ Email: smithi@maths.ox.ac.uk
}

\begin{abstract}
We study Lefschetz pencils on symplectic four-manifolds via the associated spheres in the moduli spaces of curves, and in particular their intersections with certain natural divisors. An invariant defined from such intersection numbers can distinguish manifolds with torsion first Chern class. We prove that pencils of large degree always give spheres which behave 'homologically' like rational curves; contrastingly, we give the first constructive example of a symplectic nonholomorphic Lefschetz pencil. We also prove that only finitely many values of signature or Euler characteristic are realised by manifolds admitting Lefschetz pencils of genus two curves.
\end{abstract}

AMS Classification numbers Primary: 53C15

Secondary: $57 \mathrm{R} 55$

Keywords: Lefschetz pencil, Lefschetz fibration, symplectic four-manifold, moduli space of curves

Proposed: Gang Tian

Seconded: Ronald Stern, Ronald Fintushel
Received: 7 January 2000

Revised: 13 June 2000

(C) Geometry $\&$ Topology Publications 


\section{Statements of results}

The following section shall set the investigations of this paper into a wider context, but we record the main results here for the convenience of the reader. Recall from [24] that a symplectic four-manifold gives rise to a sequence of spheres $\mathbb{S}_{X, k}^{2} \rightarrow \bar{M}_{g(k)}$ indexed by an integer $k$. The covering sequence of $X$ is the sequence of rational intersection numbers $\left(\mathbb{S}_{k}^{2} \cdot D_{k}\right)_{k \in 2 \mathbb{Z}}$. Here $D_{k}^{1} \subset \bar{M}_{2 k-1}$ is a rational multiple of the divisor of curves which are $k$-fold covers of $\mathbb{P}^{1}$, lifted to $D_{k} \subset \bar{M}_{2 k-1, h(k)}$ and translated by a term $\psi_{h(k)}$. The precise definition is given in (3.3). Suppose that $X$ is a symplectic manifold whose minimal model is not rational or ruled.

(1.1) Theorem If the covering sequence of $X$ is bounded above then $2 c_{1}(X)$ $=0$. It vanishes identically iff $2 c_{1}(X)=0=c_{2}(X)$.

This is a disguised version of results of Taubes and others on the sign of $K_{X} \cdot \omega$ for most symplectic manifolds. The bulk of the proof involves showing that the relevant Lefschetz pencils contain no reducible fibres, which is a vanishing result for certain intersection numbers of the sphere in moduli space.

(1.2) Corollory For any symplectic manifold $X$ which is not rational ruled, and pencils of high degree $k$ on $X$, the sphere $\mathbb{S}_{X, k}^{2}$ meets all known effective divisors algebraically positively.

In this sense the spheres are "homologically rational". The results for small rather than asymptotically large degree $k$ are more satisfactory.

(1.3) Theorem There is a symplectic genus three Lefschetz pencil which is not holomorphic.

In fact the positive relation (4.9) lifts to the once marked mapping class group and defines such a pencil. This is the first existence proof for symplectic nonholomorphic pencils independent of Donaldson's theorem. All previous (explicit) examples of symplectic non-Kähler Lefschetz fibrations arose from fibre sum operations and admitted no sections of square $(-1)$. We do not establish whether the total space is in fact Kähler; it is homeomorphic to a complex surface of general type. Moreover we have:

(1.4) Theorem Only finitely many pairs $\left(c_{1}^{2}, c_{2}\right)$ are realised by the total spaces of genus two Lefschetz pencils. 
This is false for Lefschetz fibrations of genus two. It would be a non-trivial consequence of the symplectic isotopy conjecture of Siebert and Tian, for which it therefore adduces additional evidence. It remains a very interesting question to investigate corresponding finiteness statements for the geography of manifolds with pencils of higher genus.

Acknowledgements Thanks to Denis Auroux for comments on an earlier draft of this paper and to Terry Fuller for permission to reproduce one of his examples. This material formed the basis of a talk at the 2000 Gokova Conference on Geometry and Topology - I am grateful to the organisers and participants for a very enjoyable conference. Special thanks to Justin Sawon for help taming tortoises.

\section{Introduction}

A fundamental problem in four-dimensional topology is to establish the relationship between arbitrary smooth four-manifolds and symplectic four-manifolds on the one hand, and between symplectic four-manifolds and Kähler surfaces on the other. To this end, the following questions are natural:

(2.1) Question (1) Given an almost complex four-manifold $X$ and $\alpha \in$ $H^{2}(X ; \mathbb{R})$ of positive square, does $\alpha$ contain symplectic forms inducing the given almost complex structure?

(2) If the symplectic manifold $X$ is homeomorphic to a Kähler surface, is the symplectic form isotopic to a Kähler form?

(3) If $X$ is Kähler and $C \subset X$ is a symplectic submanifold realising a homology class with smooth complex representatives, is $C$ itself isotopic to a complex curve?

Negative examples for each of these questions are known, largely following work in gauge theory. However, until recently no other techniques had yielded comparable progress. (The appeal of insight from other arenas is clear, given the continuing mystery of the analogous questions in higher dimensions.) In the papers [5] and [22], Simon Donaldson and the author apply the machinery of Lefschetz pencils on symplectic manifolds to construct symplectic submanifolds, reproving some results of Taubes. In particular this leads again to negative examples for the first question. This paper describes one unsuccessful attempt 
to use Lefschetz pencils to answer the second and third questions. Despite the lack of success, we believe the methods are of interest.

The paper is essentially a sequel to [24] but to be self-contained we begin with a few background notions.

(2.2) Definition A Lefschetz pencil $f: X \rightarrow \mathbb{S}^{2}$ on a four-manifold $X$ comprises a map $f$ from the complement of finitely many points $p_{i}$ in $X$ to the two-sphere, with finitely many critical points $q_{j}$, all in distinct fibres, such that

(1) $f$ is locally quadratic near all its critical points $q_{j}$ : there are local complex co-ordinates with respect to which the map takes the form $\left(z_{1}, z_{2}\right) \mapsto z_{1} z_{2}$;

(2) $f$ is locally Hopf near all its base-points $p_{i}$ : there are local complex coordinates with respect to which the map takes the form $\left(z_{1}, z_{2}\right) \mapsto z_{1} / z_{2}$.

Moreover all the local complex co-ordinates must agree with fixed global orientations.

It follows that the four-manifold is symplectic (we will say more about the cohomology class of the symplectic form below, cf 2.9 ). In projective geometry the appearance of Lefschetz pencils is classical; a generic pencil of divisors on a complex surface gives rise to such a structure, and blowing up the base-points yields a "Lefschetz fibration". More recently the inspirational work of Donaldson [6] has extended the techniques and the descriptions to the symplectic category:

(2.3) Theorem (Donaldson) Let $(X, \omega)$ be an integral symplectic manifold and let $\mathcal{L}_{\omega}$ denote the line bundle with first Chern class $[\omega]$.

(1) For $k \gg 0$ there exist pairs $\left(s_{1}, s_{2}\right)$ of approximately holomorphic sections of $\mathcal{L}_{\omega}^{\otimes k}$ such that the map $X \backslash\left\{s_{1}=s_{2}=0\right\} \rightarrow \mathbb{P}^{1}$ may be perturbed to define a Lefschetz pencil.

(2) The fibres of the pencil are symplectic submanifolds (away from the finite set of critical points) representing the Poincaré dual of $k[\omega]$ in $H_{2}(X, \mathbb{Z})$.

(3) Once $k$ is sufficiently large, the pencils obtained in this way are canonical up to isotopy.

If we blow up the base-points $p_{i}$ of a pencil, the map $f$ extends to the total space and we obtain a Lefschetz fibration. The generic fibre of the fibration is a smooth two-manifold $\Sigma_{g}$ of some fixed genus $g$; in this paper $g \geq 2$ unless stated otherwise. 
(2.4) Remark On notation We will use the term "fibre" to refer to the generic hypersurface in a pencil, as well as the preimage of a point in a fibration. Note that we reserve the term "pencil" to refer to the four-manifold before blowing up, and so the fibres in a pencil have strictly positive square.

The topology of the four-manifold $X$ is encoded in a positive relation; this is a word $\left\langle\prod \delta_{i}=1\right\rangle$ in positive Dehn twists in the mapping class group $\Gamma_{g}$ which determines the monodromy representation and the diffeomorphism type of the fibration. Here $\delta_{i}$ is the positive Dehn twist about some fixed (isotopy class of) embedded curve $C_{i} \subset \Sigma_{g}$; occasionally we shall use $\delta$ for the curve as well as the twist diffeomorphism. Such curves are called vanishing cycles and generate a subgroup $V<\pi_{1}\left(\Sigma_{g}\right)$, which we always assume is non-empty. The fundamental group of the four-manifold $X$ is given by $\pi_{1}\left(\Sigma_{g}\right) / V$. All of our vanishing cycles $C$ will be homotopically essential and hence the fibres will contain no spherical components. Given this, if we choose a metric on $X$ the smooth fibres become Riemann surfaces and the critical fibres stable Riemann surfaces, and we induce a map $\phi_{f}: \mathbb{S}^{2} \rightarrow \bar{M}_{g}$ with image the Deligne-Mumford moduli space of stable curves. Recall this moduli space is given by adjoining certain divisors of stable curves $\Delta_{i}(0 \leq i \leq[\mathrm{g} / 2])$ to the moduli space of smooth curves $M_{g}$. The $\Delta_{i}$ form the irreducible components of a connected divisor $\Delta=\cup \Delta_{i}$ and the generic curve in $\Delta_{i}$ has one component of genus $i$ and one of genus $g-i$ if $i>0$, and is irreducible if $i=0$. The fibration being symplectic means that the sphere $\mathbb{S}_{X}^{2}$ has locally positive intersections with the various divisors $\Delta_{i}$; the restriction to nodal singularities means the intersections are transverse. In this paper we shall refer to a fibration $f$ on $X$ giving rise to a sphere $\mathbb{S}_{X}^{2}$ and will suppress the choice of metric; changing the metric changes the sphere by an isotopy which always preserves the geometric intersection number with the $\Delta_{i}$ (an "admissible isotopy"). All results depend only on the admissible isotopy class of the sphere.

(2.5) Remark In [5] and [22], symplectic submanifolds are constructed by studying the Gromov invariants of fibrations of symmetric products associated to a Lefschetz pencil. In [20] Gromov invariants for associated families of moduli spaces of stable bundles are related to instantons on the four-manifold. However, the spheres $\mathbb{S}^{2} \rightarrow \bar{M}_{g}$ in this paper are not necessarily symplectic or pseudoholomorphic. It remains an interesting question to study the quantum cohomology of moduli spaces of curves.

The geometric classes $\Delta_{i}$ define elements of $H^{2}\left(\bar{M}_{g} ; \mathbb{Z}\right)$; there is an important algebraic class which is not dual to any distinguished divisor. This is the Hodge 
class $\lambda$, which is the first Chern class of the relative dualising sheaf of the universal curve $\mathcal{C}_{g} \rightarrow M_{g}$. Although the universal curve does not exist, the Chern class makes sense. There are additional natural classes when we look at moduli spaces of curves with (ordered) marked points. Let $\psi_{i}$ be the first Chern class of the line bundle on $\bar{M}_{g, h}$ with fibre $T_{p_{i}}^{*}$ at $\left(\Sigma ; p_{1}, \ldots, p_{h}\right)$. We record the following, which is due to Harer [10] and Arbarello-Cornalba [1]:

(2.6) Theorem (Harer, Arbarello-Cornalba) The classes $\Delta_{i}$ with $0 \leq i \leq$ $[g / 2]$ and $\lambda$ rationally generate the second cohomology $H^{2}\left(\bar{M}_{g} ; \mathbb{Q}\right)$. For the pointed moduli space, $H^{2}\left(\bar{M}_{g, h} ; \mathbb{Q}\right)$ is rationally generated by the pullbacks of these classes under the forgetful map and the classes $\left\{\psi_{i} \mid 1 \leq i \leq h\right\}$.

The two descriptions of a four-manifold given by a positive relation and a sphere in moduli space seem rather far from one another. Nonetheless, they may be related, and we shall exploit the dual descriptions in investigating the consequences of the following trivial result:

(2.7) Proposition If $f: X \rightarrow \mathbb{S}^{2}$ gives rise to a sphere $\mathbb{S}^{2} \subset \bar{M}_{g}$ and for some effective divisor $D \subset \bar{M}_{g}$ not containing $\mathbb{S}^{2}$ we have $\left[\mathbb{S}^{2}\right] \cdot[D]<0$ then $f$ is not isotopic to a holomorphic fibration.

This suggests a natural obstruction to the existence of Kähler forms isotopic to given symplectic forms:

(2.8) Corollory Let $(X, \omega)$ be a symplectic manifold and suppose the Lefschetz pencils obtained from asymptotically holomorphic sections of $\mathcal{L}_{\omega}^{\otimes k}$ give spheres $\mathbb{S}_{k}^{2} \subset \bar{M}_{g(k)}$. If for some sequence of divisors $D_{k} \subset \bar{M}_{g(k)}$ we have that $\mathbb{S}_{k}^{2} \not \subset D_{k}$ and $\left[\mathbb{S}_{k}^{2}\right] \cdot D_{k}<0$ then $\omega$ is not deformation equivalent to a Kähler form on $X$.

Proof According to Donaldson [6] the pencils defined by his construction from approximately holomorphic sections are isotopic to the pencils provided by algebraic geometry for $k \gg 0$. These pencils give rise to rational curves in moduli space, which must meet positively all effective divisors in which they are not contained. Thus the assumptions indicate that the spheres defined by the given manifold $X$ are not isotopic to rational curves. By the asymptotic uniqueness in Donaldson's theorem, this shows that the symplectic structure on $X$ is not in fact Kähler. 
It is important to note that these obstructions might well be computable. Given one explicit positive relation (with base-points) defining a pencil, there is a stabilisation process which obtains pencils of higher degree [3]. If the initial pencil is obtained from approximately holomorphic sections, so are the later ones. Moreover, there are familiar techniques for computing cohomology classes of divisors in moduli space and hence the intersection numbers above, whilst the condition that the sphere lies inside a particular divisor may have topological consequences which can be checked independently. We shall use such an argument in the proof of (1.3) later in the paper. Nonetheless, our main observation - contained in the theorems (1.1) and (1.2) - amounts to the triviality of these obstructions. In principle it remains possible that the obstructions could detect symplectic manifolds with $2 c_{1}=0$ which were not Kähler, but this seems implausible.

In the face of these results, we shall turn from studying the asymptotic intersection behaviours to concentrating on rather particular divisors at small genus; in this framework we shall deduce the results (1.3) and (1.4). The first instance involves the hyperelliptic divisor inside the moduli space of genus three curves. In particular, we show that in certain circumstances one can detect from a positive relation the non-holomorphicity of a fibration, even when the total space is homotopy Kähler. The finiteness result will rely on working with pairs comprising a Lefschetz fibration and a distinguished section, and with a certain Weierstrass divisor in the moduli space of pointed genus two curves. We leave the details until the relevant discussion in the paper. We do draw attention to one general fact however. The first constructions of symplectic structures on manifolds with Lefschetz pencils involved blowing up to the Lefschetz fibration, and blowing down exceptional symplectic sections again. This led to an unfortunate asymmetry: Donaldson's theorem (2.3) gives Lefschetz pencils whose fibres are dual to a given integral symplectic form, but given a Lefschetz pencil there may be no symplectic form in the integral cohomology class which is dual to a fibre.

(2.9) Lemma There are smooth four-manifolds $X$ with the topological structure of Lefschetz pencils for which the class $P D[$ Fibre $] \in H^{2}(X ; \mathbb{Z})$ admits no symplectic representative symplectic on the fibres.

Proof Let $X$ be a manifold with a pencil of curves with one base-point, and for which some member of the pencil is a reducible curve. Such can be obtained by blowing up all but one of the base-points on any pencil containing reducible elements, for instance the pencil obtained by Matsumoto [14]. Suppose for contradiction that there is a symplectic form in the class $P D[$ Fibre] for which the 
smooth locus of each fibre is a symplectic submanifold. Then each component of a reducible fibre has positive symplectic area, and hence since the symplectic form is integral $[\omega] \cdot[$ Fibre $] \geq 2$. But we have assumed there is a unique base-point.

This is the only source of such pathologies: according to a recent theorem of Gompf [9], if there are base-points on every component of every fibre then there is a symplectic form in the distinguished integral class. It follows that the nonKähler pencil that we construct will indeed determine a canonical isotopy class and not deformation equivalence class of symplectic form on the four-manifold.

We note in closing that there is an analogue of the foundational (2.7) which applies to branched coverings:

Suppose $\overline{\mathcal{N}}_{g} \rightarrow \bar{M}_{g}$ is a branched covering. Then a sphere $\mathbb{S}^{2} \subset \bar{M}_{g}$ lifts to the covering space $\overline{\mathcal{N}}_{g}$ if and only if it meets the branch locus everywhere tangentially.

There is an obvious topological constraint which must be satisfied if such tangential intersections can arise: the intersection number between the sphere and the branch divisor must be even. This leads to a circle of ideas somewhat similar to that developed in this paper.

\section{$3 \quad$ Asymptotic intersections}

In this section we shall prove the result (1.1) and explain the shape of the statement (1.2). There is a well-known conjecture on the geometry of the moduli spaces $\bar{M}_{g}$. Recall Harer's theorem from above; the following is taken from [11].

(3.1) Conjecture "Slope Conjecture", Harris-Morrison Let $a \lambda-b \Delta_{0}$ be represented by an effective divisor in $\bar{M}_{g}$. The ratio $a / b$ is minimised by Brill-Noether divisors.

This special property of Brill-Noether divisors ${ }^{1}$ motivates the definition of a particular symplectic invariant. By the adjunction formula, the pencils of even degree $2 k$ on any symplectic manifold give rise to families of curves of odd genus. In these moduli spaces we have divisors $D_{(g+1) / 2}^{1} \subset \bar{M}_{g}$ comprising the

\footnotetext{
${ }^{1}$ That is, divisors of curves which have a linear system they shouldn't have.
} 
codimension one components of the closure in $\bar{M}_{g}$ of the locus of curves in $M_{g}$ which admit a $g_{(g+1) / 2}^{1}$. (Recall that a $g_{d}^{r}$ is a linear system of dimension $r$ and degree $d$.) Since a linear system of dimension one and degree $d$ is just a $d$-fold covering over $\mathbb{P}^{1}$, the hyperelliptic divisor is precisely $D_{2}^{1} \subset \bar{M}_{3}$. For even $g$ there are analogous "Petri" divisors, but we will avoid the notational complication they introduce.

(3.2) Theorem (Harris-Mumford) Let $g$ be odd and $k=(g+1) / 2$. The cohomology class of $D_{k}^{1}$ is given by

$$
D_{k}^{1}=c_{k}\left((g+3) \lambda-\frac{g+1}{6} \Delta_{0}-\sum_{i=0}^{[g / 2]} i(g-i) \Delta_{i}\right) .
$$

Here $c_{k}=3(2 k-4) ! /(k !(k-2) !)$ is positive and rational.

It shall be important for us to normalise the Brill-Noether divisors by dividing by these rational constants $c_{k}$. A Lefschetz pencil with $h$ base-points gives rise to a sphere in a moduli space $\bar{M}_{g, h}$ of curves with $h$ ordered marked points. We need to keep track of the marked points in order to utilise all the geometry of the system; we do this by translating the divisor $D_{k}^{1}$ when we lift to $\bar{M}_{g, h}$.

(3.3) Definition Let $(X, \omega)$ be an integral symplectic manifold. The covering sequence of $(X, \omega)$ is the sequence of intersection numbers $\left(\left[\mathbb{S}_{k}^{2}\right] \cdot D_{k}\right)_{k \in 2 \mathbb{N}}$ between the spheres defined by pencils of curves dual to $k \omega$ on $X$ and the divisors

$$
D_{k}=\frac{1}{c_{k}} D_{k}^{1}-\sum_{j=1}^{k^{2} \omega^{2}} \psi_{j}
$$

We set the intersection number to be zero by convention if there is no pencil of curves in the relevant homology class, or if $g=g(k)$ is not odd.

If two integral symplectic manifolds $\left(X, \omega_{X}\right)$ and $\left(Y, \omega_{Y}\right)$ are symplectomorphic then it follows from the theorem of Donaldson (2.3) that their covering sequences co-incide for sufficiently large $k$. The values of the covering sequence compare the numbers of exceptional (for instance hyperelliptic) fibres in the Lefschetz fibration, normalised by the universal constants $c_{k}$, with the number of exceptional sections of the fibration. This is quite a natural comparison: at sufficiently large $k$ we know the pencils of approximately holomorphic sections can be extended to nets (two dimensional linear systems) of sections [2]. A net gives a branched cover of $X$ over $\mathbb{C P}^{2}$, or equivalently a branched cover of 
the total space of a Lefschetz fibration over the first Hirzebruch surface $\mathbb{F}_{1}$ in which all the exceptional sections of the Lefschetz fibration map to the unique holomorphic section of square $(-1)$ in $\mathbb{F}_{1}$. Hence for nets to exist, we know that the fibres of the Lefschetz pencil must admit branched coverings over $\mathbb{P}^{1}$ of the explicit degree given by the number of base-points. Thus we are comparing this degree valid for all the fibres to the minimal degree realised by some of them.

To establish a link between four-manifolds and intersection numbers, we shall need to understand the values of the generators of $H^{2}$ on a sphere $\left[\mathbb{S}^{2}\right]$. For the $\Delta_{i}$ it is straightforward - we count the numbers of singular fibres of different kinds. The main result of [24] solves the problem for the Hodge class, which is related to the signature of the associated Lefschetz fibration:

$$
\sigma(X)=4\left\langle\lambda,\left[\mathbb{S}_{X}^{2}\right]\right\rangle-\sum\left\langle\Delta_{i},\left[\mathbb{S}_{X}^{2}\right]\right\rangle .
$$

We have an easy lemma:

(3.5) Lemma Let $X \rightarrow \mathbb{S}^{2}$ be a Lefschetz fibration with a distinguished section $s$. Then the value $\left\langle\psi,\left[\mathbb{S}_{X, s}^{2}\right]\right\rangle$ of $\psi \in H^{2}\left(\bar{M}_{g, 1} ; \mathbb{Z}\right)$ on the associated sphere is given by the self-intersection $s \cdot s$.

Lastly recall the adjunction formula; for a pencil of curves on $X$ dual to $k[\omega]$ we have

$$
2 g-2=K_{X} \cdot k[\omega]+k^{2}[\omega]^{2} .
$$

From these pieces of information, and the result (3.2), we can now derive all the values of the intersections $\mathbb{S}_{k}^{2} \cdot D_{k}$ from a combinatorial description of a Lefschetz fibration. However, the topological type of the manifold does not suffice, largely because it is not clear how to determine the individual values $\Delta_{i}$ rather than their sum. In fact, if $i>0$ we can expect the value to be zero. The motivation comes from complex geometry:

(3.7) Proposition Suppose the Kähler surface $X$ has a Lefschetz pencil of curves in a homology class $[C]$. If the pencil contains a reducible curve, then either $[C]$ is indivisible in $H_{2}(X, \mathbb{Z})$ or $[C]=2[D]$ with $[D]$ indivisible and $[D]^{2}=1$.

Proof Recall that the Hodge Index theorem asserts that the intersection form on $H^{1,1}$ has a unique positive eigenvalue for any Kähler surface. In particular, given any divisor $D$ for which $D^{2}>0$ and a divisor $D^{\prime}$ such that $D \cdot D^{\prime}=0$ 
we can deduce that either $D^{\prime}=0$ or $D^{\prime} \cdot D^{\prime}<0$. We apply this in the following way. First note, scaling $k$ if necessary, we have in the notation of (3.9) that $\left(D_{1}\right)^{2}+\left(D_{2}\right)^{2}=k^{2}[C]-2>2$, and we may assume without loss of generality that $\left(D_{1}\right)^{2}>0$. Now

$$
D_{1} \cdot\left(D_{1}-\left(D_{1}\right)^{2} D_{2}\right)=0 \Rightarrow D_{1}=\left(D_{1}\right)^{2} D_{2} \text { or }\left(D_{1}-\left(D_{1}\right)^{2} D_{2}\right)^{2}<0 .
$$

The first case gives an easy contradiction. If $D_{1}=\left(D_{1}\right)^{2} D_{2}$ then $D_{1} \cdot D_{2}=$ $\left(D_{1}\right)^{2}\left(D_{2}\right)^{2}=1$ which forces $\left(D_{1}\right)^{2}=\left(D_{2}\right)^{2}=1$ by integrality; but this is impossible, since $\left(D_{1}\right)^{2}+\left(D_{2}\right)^{2}>2$. So assume instead that the second case holds, and expanding we find that $\left(D_{1}\right)^{2}\left[\left(D_{1}\right)^{2}\left(D_{2}\right)^{2}-1\right]<0$ which gives $\left(D_{1}\right)^{2}\left(D_{2}\right)^{2}<1$, and hence $\left(D_{2}\right)^{2} \leq 0$ since the first of the two terms is assumed positive. Now since

$$
k \mid\left(D_{2} \cdot[k \omega]\right)=D_{2}\left(D_{1}+D_{2}\right)=1+\left(D_{2}\right)^{2}
$$

we know that we cannot have $\left(D_{2}\right)^{2}=0$ as soon as $k$ is not equal to 1 . But now we have a contradiction again: by assumption, each of the $D_{i}$ appeared as complex curves in a pencil and hence has positive symplectic area. From which

$$
[k \omega] \cdot D_{2}>0 \Rightarrow 1+\left(D_{2}\right)^{2}>0
$$

which is absurd if also $\left(D_{2}\right)^{2} \leq-1$. Thus as soon as $k$ is large enough that $k^{2}[C]^{2}>4$ and $k>1$, no Lefschetz pencil of curves in the class $[C]$ on a Kähler surface can contain reducible elements.

There are analogues of this result for general symplectic pencils. With the stabilisation procedure for pencils in mind, we shall concentrate on pencils of even degree. Then there is a trivial argument for certain classes of fourmanifold, even without the Kähler assumption:

(3.8) Proposition Suppose the symplectic manifold $X$ has even intersection form, or that $K_{X}$ is two-divisible in cohomology. Then a Lefschetz pencil dual to $2 k[C]$, for any $k \in \mathbb{Z}_{+}$and $[C] \in H_{2}(X ; \mathbb{Z})$, contains no reducible fibres.

Proof To obtain a reducible curve in a pencil of curves dual to $k[C]$ precisely involves writing

$$
k[C]=D_{1}+D_{2} \in H^{2}(X, \mathbb{Z}) \quad \text { where } \quad D_{1} \cdot D_{2}=1 .
$$

But this is impossible under the additional assumptions on $X, k$; for if $D_{1}=$ $r[C]+\alpha$ and $D_{2}=(k-r)[C]-\alpha$ then

$$
D_{1} \cdot D_{2}=r(k-r)[C]^{2}+(k-2 r)[C] \cdot \alpha-\alpha^{2} .
$$


But if $k$ is even and the intersection form is even, all three terms are divisible by two and hence the LHS cannot equal 1. Using another trick, we see that if $K_{X}$ is even (ie, can be written as $2 \kappa$ for some cohomology class $\kappa$ ) then by adjunction $\left(D_{1}\right)^{2}$ must be even:

$$
2 g_{D_{1}}-2=K_{X} \cdot D_{1}+\left(D_{1}\right)^{2}
$$

but on the other hand, for even $k$, we have

$$
D_{1} \cdot(k[C])=D_{1} \cdot\left(D_{1}+D_{2}\right)=\left(D_{1}\right)^{2}+1
$$

forcing $\left(D_{1}\right)^{2}$ to be odd, a contradiction.

Nonetheless, to obtain the general case requires more work. The following can be regarded as a symplectic shadow of the Hodge Index theorem. Note that it is in fact a vanishing result; it implies that the intersection numbers $\left[\mathbb{S}_{k}^{2}\right] \cdot \Delta_{i}=0$ whenever $k$ is large and even, and $i>0$. A detailed algebraic treatment of stabilisation is now available in [3].

(3.10) Theorem Every symplectic four-manifold admits Lefschetz pencils composed of only irreducible curves. Indeed the pencils arising from the stabilisation $k \mapsto 2 k$ procedure always have this form.

Proof The central observation we need is due to Donaldson; it is easier to pass from Lefschetz pencils representing $k \omega$ to ones representing $2 k \omega$ than to ones representing $(k+1) \omega$. Suppose then we have a symplectic four-manifold $X$ and a pencil of sections of a line bundle $L$ with $c_{1}(L)=k \omega$ (normalised appropriately). Let the pencil of sections $\left\{s_{1}+\lambda s_{2}\right\}_{\lambda \in \mathbb{P}^{1}}$ be generated by two smooth elements $s_{1}, s_{2}$. We consider the pencil of reducible nodal sections $\left\{s_{1}^{2}+\lambda s_{1} s_{2}\right\}_{\lambda \in \mathbb{P}^{1}}$. Thus we "add in" the zero-set of $s_{1}$ to each of the curves in the original pencil. In complex geometry this would correspond to taking a sphere $\mathbb{P}^{1} \subset \mathbb{P} H^{0}(L)$ with image entirely contained in the discriminant locus of singular curves. Such a sphere could be perturbed to have isolated transverse intersections with the discriminant, corresponding to a deformation to the Lefschetz situation; the above remarks would then apply. We mimic this by (the first rather easy steps of) an analysis of the relevant deformation. Note that the singular sections still satisfy the approximate holomorphicity and $C$-bounded constraints of [6] for suitable constants, and it is transversality that we must achieve.

Away from $(\lambda=0)$ in the $\lambda$-plane, we have a family of nodal curves parametrised by a disc. There is a small $C^{2}$-deformation of this family, analogous to 
smoothing a single nodal curve into the self-connect sum of its normalisation. Thus a perturbation of the form

$$
\left\{\left(s_{1}^{2}+\epsilon\right)+\lambda\left(s_{1} s_{2}+\delta\right)\right\}_{|\lambda| \gg|\epsilon|+|\delta|}
$$

smooths each of the curves $s_{1}^{2}, s_{1} s_{2}$ and for generic smoothing sections $\epsilon, \delta$ will give at least away from $\lambda=0$ a Lefschetz family by the arguments of [6]. But by continuity we can see the critical members of this family: they arise (again for large $|\lambda|$ ) in one-to-one correspondence with the critical fibres of the monodromy of the original pencil $\left\{s_{1}+\lambda s_{2}\right\}$ on $X$.

It follows that the new monodromy near infinity in the $\lambda$-plane gives a copy of the old monodromy in a model where we identify each original fibre $\left(s_{2}=\right.$ constant) minus small discs with its image in a smoothed fibre. The product of the original Dehn twist monodromies is no longer the identity but equal to a word in the (commuting) Dehn twists about the necks that link a copy of $s_{2}$ with a copy of $s_{1}$ once the finitely many nodal intersections are smoothed. Thus we must determine an integer $n_{i}$ for each of these nodes, and the monodromy about a large circle around 0 in the $\lambda$-plane is given by $\prod \delta_{i}^{n_{i}}$ where $i$ runs over the base-points of the original pencil. By symmetry each of the $n_{i}$ will be equal, since this is a local question: we are taking a section of a line bundle over $\mathbb{P}^{1}$ with fibre the tensor product of the tangent directions at the node. (This can be identified with the normal bundle to the divisor of stable nodal curves in the moduli space $\bar{M}_{g}$.) Since the tangent direction at the fixed curve is constant, the line bundle is the normal bundle to the section defined by the base-point. Since the exceptional sections have normal bundle $\mathcal{O}(-1)$ we deduce that $n_{i}=-1 \forall i$.

The situation is now as depicted in Figure 1. The remaining critical fibres in the $2 k$-pencil come from deforming the multiple fibre $s_{1}^{2}=0$ to a smooth locus. This is again a local consideration and we can proceed in various ways. If $b_{+}(X)>1$ we can choose an integrable complex structure in any sufficiently small neighbourhood of the multiple fibre. This involves identifying to diffeomorphism the model with a neighbourhood of a multiple fibre in a pencil of singular curves on a Kähler surface - note that the local diffeomorphism type depends only on the genus of the curve and the number of base-points of the original pencil. On the other hand, for the Kähler situation reducible curves of Lefschetz type cannot occur for topological reasons, as described above. Thus we need to know that there is always a Kähler model available. If the fourmanifold has $b_{+}>1$ this follows from work of Taubes [27]: we know that the number of base-points is bounded above by $2 g-2$ (by the positivity of $K \cdot \omega$, cf 5.4). But then take a genus $g$ pencil on a holomorphic $K 3$ surface; this 


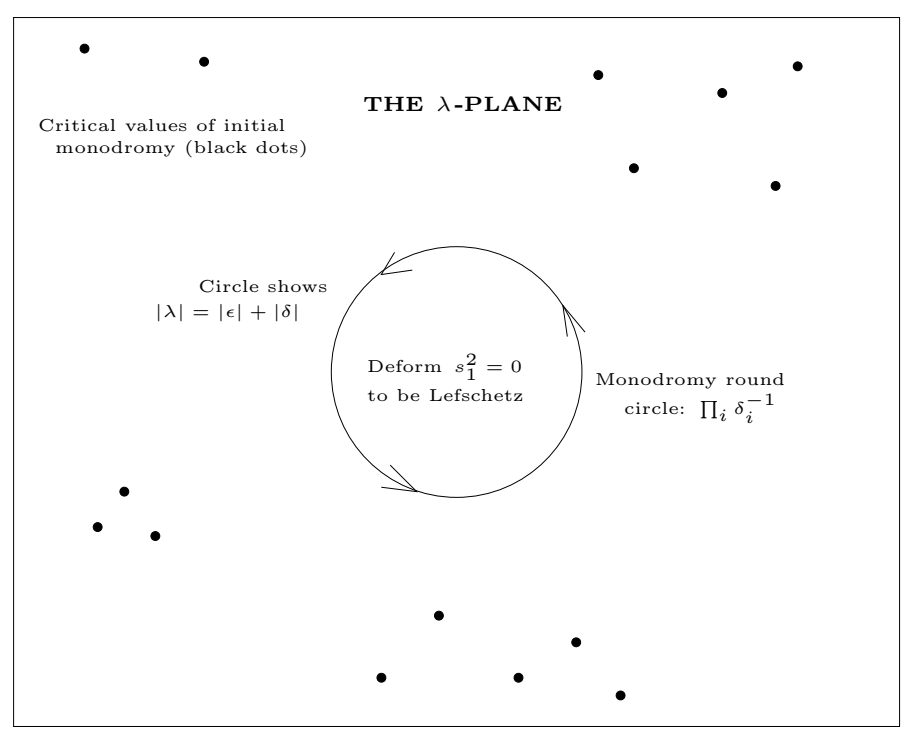

Figure 1: Stabilisation viewed in the $\lambda$-plane

has $2 g-2$ base-points, so blowing these up successively gives pencils of curves with any strictly positive intermediate number of base-points. Moreover the singular holomorphic pencils do have Lefschetz smoothings, by Riemann-Roch; in any high dimensional linear system of curves with some smooth members, the generic pencil is Lefschetz by Bertini's theorem and complex Morse theory. For large $k$ we can assume that our divisors are very ample and smooth curves do exist.

To avoid the Seiberg-Witten theory, or when $b_{+}=1$, we can study the local degeneration directly. For instance, fix a family of smooth curves with an arbitrary number $N$ of sections over a disc. Gluing on a copy of the central fibre along the sections gives a disc in a moduli space $\bar{M}_{g, N}$ which is contained entirely in the stable locus. By projectivity of this stable divisor, there is some complex curve in the stable locus which contains an isotopic copy of some sufficiently small sub-disc; then a holomorphic perturbation of this complex curve will be transverse to the stable locus and will also give a model for the relevant local degeneration. In another direction, we could model the $k \mapsto 2 k$ stabilisation by a perturbation

$$
\left\{s_{1}+\lambda s_{2}\right\}_{\lambda \in \mathbb{P}^{1}} \Rightarrow\left\{\left(s_{1} s_{3}+\epsilon\right)+\lambda\left(s_{2} s_{3}+\delta\right)\right\}_{\lambda \in \mathbb{P}^{1}} .
$$

Here we obtain the section $s_{3}$ from Auroux's construction of nets [2]. By 
uniqueness at large enough $k$, this pencil must be isotopic to that given by the previous stabilisation, and the distinct local models imply that the smoothing of the double fibre yields no reducible vanishing cycles.

The proof is completed with a familiar trick. For the reducible fibres in the original pencil, both components are smooth symplectic submanifolds themselves. It follows that for each component $D_{i}$ we have $(\omega) \cdot D_{i}>0$ which means that there are base-points of the original pencil on both components. Once we add in a copy of $s_{1}$ and smooth the nodes, this precisely means that the separating vanishing cycles no longer separate the new generic fibre. Thus the separating vanishing cycles from the old monodromy become non-separating, and the stabilisation is elsewhere modelled on a complex situation in which there are no reducible curves. The theorem follows.

It may be of independent interest to observe that there is a stabilisation procedure in which the vanishing cycles at level $k$ form a subset of a natural set of vanishing cycles at level $2 k$. Note that we could not make sense of this without considering the base-points of the pencil as well as the vanishing cycles of the fibration. We can now prove the first theorem (1.1).

(3.11) Theorem Suppose that $X$ is not rational or ruled. The covering sequence of $X$ is bounded above only if $2 c_{1}(X)=0$, and vanishes identically iff $2 c_{1}(X)=0, c_{2}(X)=0$.

Proof We compute the intersection number $\left[\mathbb{S}_{k}^{2}\right] \cdot D_{k}$ using the formulae $(3.2$, $3.4,3.6)$ as well as the definition (3.3) and the vanishing theorem (3.10). The classes $\psi_{i}$ evaluate, by the lemma (3.5), on any sphere arising from a pencil to give -1 , and hence we can compute the intersection with $D_{k}^{1} / c_{k}$ and then adjust by subtracting the number of base-points of the pencil. Of course this is just $k^{2} \omega^{2}$. The calculation gives

$$
\left[\mathbb{S}_{k}^{2}\right] \cdot D_{k}=\frac{(g+7)(g+1)}{12} K_{X} \cdot \omega+\frac{g+3}{12}\left(c_{1}^{2}(X)-c_{2}(X)\right)-\frac{2}{3} c_{2}(X) .
$$

Here $\omega$ refers to the given integral symplectic form on $X$ and not some multiple, and we have substituted $g=2 k-1$. This expression will grow positively as $o\left(g^{2}\right)$ unless $K_{X} \cdot \omega \leq 0$. But we know from Seiberg-Witten theory [12], [15] that $K_{X} \cdot \omega<0$ only if $X$ is a rational or ruled surface, which we exclude by assumption, and that $K_{X} \cdot \omega=0$ only if $2 K_{X}=0$. If $b_{+}>1$ then we deduce that $K_{X}=0$ itself.

If $K_{X}$ is a torsion class, then the intersection number is given by a negative multiple of $c_{2}(X)$ and hence the covering sequence is identically zero iff this 
also vanishes. The last thing we need to know is that when $K_{X}$ is torsion, $c_{2}(X) \geq 0$ so that the sequence is necessarily bounded above in this case. But if $2 c_{1}(X)=0$ and $c_{1}(X) \neq 0$ then we know that $b_{+}(X)=1$, and then the result follows since $0=c_{1}^{2}=2 c_{2}+3\left(1-b_{-}\right)$. (It is likely, but not proven in general, that whenever $c_{1}(X)=0$ then $c_{2}(X) \geq 0$; this would give "iff" in the first statement of the Theorem.)

The intersection formula above was twisted by subtracting the large number of exceptional sections from the naive intersection $\left.\left[\mathbb{S}_{k}^{2}\right] \cdot\left[\left(1 / c_{k}\right) D_{k}^{1}\right]\right)$. Given the slope conjecture, we see that for $K_{X} \cdot \omega>0$ the intersection numbers of spheres in moduli space with any effective divisor grow unbounded to infinity. Indeed this growth is quadratic with the degree $k$. This justifies the corollary (1.2), and explains the failure of the obstructions (2.8) to distinguish symplectic and Kähler structures in four dimensions.

(3.12) Remark If we have a Kähler surface with $K_{X} \cdot \omega<0$ then we have shown that the spheres defined by pencils of large enough degree will necessarily be contained in all of the Brill-Noether divisors. This however is not so surprising. Recall that for $g>23$ the moduli space $\bar{M}_{g}$ is known to be of general type and not unirational (as the first few moduli spaces are, when $g \leq 11$ ). It follows that through the general point of $\bar{M}_{g}$ there is no rational curve, and all the spheres defined by holomorphic fibrations lie inside distinguished subvarieties of special curves (with no condition on $K \cdot \omega$ of the underlying surface).

In [24] we proved that the symplectic area of the spheres $\mathbb{S}_{k}^{2}$ is positive for any symplectic manifold $X$ and pencils of any degree $k$. This follows from the statement that the evaluation $\left\langle\lambda,\left[\mathbb{S}^{2}\right]\right\rangle$ is strictly positive. In the light of the above, it makes sense to ask if the principle (2.7) can ever be applied; can any sphere meet any effective divisor negatively? Certainly local negative intersections must arise, for spheres which are not isotopic to rational curves, but it is not clear that these can ever contribute sufficiently to give a negative algebraic intersection. In the next section we shall provide an explicit example to show that (2.7) is not entirely vacuous.

\section{Symplectic non-Kähler Lefschetz pencils}

Every Kähler surface admits a holomorphic Lefschetz pencil. Is every Lefschetz pencil on a smooth four-manifold in fact holomorphic for some Kähler structure? 
The answer is clearly no, for Donaldson's theorem (2.3) provides pencils on manifolds not homotopy equivalent to complex surfaces. In this section we provide a more down to earth answer. Exotic Lefschetz fibrations have been constructed by fibre summing known holomorphic fibrations by diffeomorphisms which twist the monodromy; examples appear in the author's thesis [23]. These fibrations are on manifolds which are not complex surfaces. Examples of nonholomorphic fibrations on manifolds homeomorphic to complex surfaces were given by Fintushel and Stern, who distinguished the total spaces from Kähler surfaces using computations of Seiberg-Witten invariants [8]. Their examples were again fibre sums. We recall a theorem of Stipsicz [26]; a simpler proof is given by the author in [21]:

(4.1) Theorem If a Lefschetz fibration admits a section of square (-1) then it cannot decompose as any non-trivial fibre sum.

To build exotic Lefschetz pencils, we will use a variant of a fibre sum construction; instead of inserting a mapping class group word of the form $\prod \delta_{i}=1$ into a monodromy word, we shall insert a more balanced word $\prod \delta_{i}=\prod \tau_{j}$ for positive twists $\delta_{i}, \tau_{j}$. Thus the example has the satisfying side-effect of marrying the combinatorial and holomorphic descriptions of Lefschetz pencils.

(4.2) Example The mapping class group of a genus $g$ surface can be generated by positive Dehn twists subject to relations supported in twice-holed tori and four-punctured spheres [13]. In this presentation, only one "basic relation" equates two non-trivial products of positive twists. Let $\Sigma_{1,2}$ be a torus with two boundary circles. Write $\delta_{1}, \delta_{2}$ for the positive twists about curves parallel to the two boundary components $(\partial \Sigma)_{i}$; there is a relation

$$
\left(\tau_{u} \tau_{v} \tau_{w}\right)^{4}=\delta_{1} \delta_{2}
$$

where $\tau_{u}$ denotes the twist about the curve labelled $U$ in Figure 2, etc.

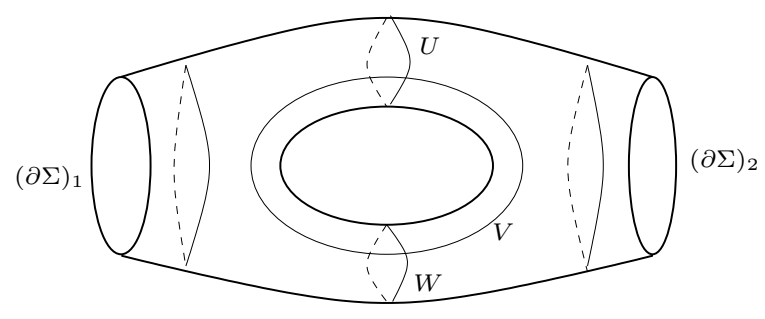

Figure 2: Supporting curves for a basic relation in $\Gamma_{1,2}$ 
Write $\mathcal{T}$ for the operation on positive relations which replaces a string $\left(\delta_{1} \delta_{2}\right)$ by the string $\left(\tau_{u} \tau_{v} \tau_{w}\right)^{4}$. An easy computation using Novikov additivity shows that $\mathcal{T}$ has the following effect on the topological invariants of the four-manifold:

$$
\sigma \mapsto \sigma-6 ; e \mapsto e+10 ; c_{1}^{2} \mapsto c_{1}^{2}+2 .
$$

These formulae must be modified if either of the boundary curves in the inserted copy of $\Sigma_{1,2}$ bounds in the higher genus surface; for instance, the signature is changed by eight and the Euler characteristic by twelve when fibre summing elliptic fibrations. Assuming we are in the generic situation, however, and for pencils with no reducible elements, that is discounting the $\Delta_{i \geq 1}$, we deduce

$$
\left[\mathbb{S}^{2}\right] \cdot(a \lambda-b \Delta) \stackrel{\mathcal{T}}{\longrightarrow}\left[\mathbb{S}^{2}\right] \cdot(a \lambda-b \Delta)-(10 b-a) .
$$

It follows that applying the operation $\mathcal{T}$ to a Lefschetz fibration provides a clean way of decreasing the intersection numbers with divisors. For if $a>11 b$ then in fact the divisor is ample and - in the vein of the remarks $(3.12)$ - we are unlikely to find any topological consequence of an inclusion $\mathbb{S}^{2} \subset D$. Thus we are most likely to deal with divisors for which $a \leq 11 b$; when also $a<10 b$ then the mapping class group insertion $\mathcal{T}$ will not increase the algebraic intersection with $D$. Moreover we have the following key lemma:

(4.4) Lemma Let $\left\langle\prod \gamma_{i}=1\right\rangle$ be a positive relation describing a Lefschetz fibration which contains an exceptional section. Assume the generic fibre genus is at least two. Then a fibration obtained by applying either $\mathcal{T}$ or $\mathcal{T}^{-1}$ to the relation also admits an exceptional section.

Proof It is enough to see that the relation $\left\langle\left(\tau_{u} \tau_{v} \tau_{w}\right)^{4} \delta_{2}^{-1} \delta_{1}^{-1}=1\right\rangle$ describes a (non-symplectic) fibration with a section of square zero. For then we can view the operations $\mathcal{T}^{ \pm 1}$ as given by fibre summing two fibrations, one with a section of square -1 and one with a section of square 0 , and then excising a piece of the fibration with trivial monodromy. This trivial piece is determined by a relation $\left\langle\left(\prod f_{i}\right)\left(\prod f_{i}\right)^{-1}=1\right\rangle$ and such relations always admit square zero sections. One can then perform the fibre sums and excisions relative to a basepoint (noting for instance that every diffeomorphism of a surface is isotopic to a once-pointed diffeomorphism) to obtain the result.

The existence of the section of square zero for the basic relation (4.2) follows from the methods of [21]. Since the genus of the generic fibre $g \geq 2$ we know that the supporting curves for the $\mathcal{T}$-relation do not fill the surface. Hence we can lift all of the individual twists to the hyperbolic disc, the universal cover of a single smooth fibre, so as to preserve some union of geodesics. Look at the 
monodromy at the circle at infinity which is the boundary of the disc; after we have lifted each (positive or negative) Dehn twist, we have lifted the identity and have a hyperbolic automorphism of the disc. But this automorphism can be taken to fix a geodesic, so must be the identity. Since the individual twists fixed points on $\mathbb{S}_{\infty}^{1}$ the total rotation number of the circle at infinity under the sequence of lifts is zero. But according to the results of [21] this precisely constructs a section of square zero.

We now return to divisors in moduli space. The first of the Brill-Noether divisors already introduced is the hyperelliptic divisor $\mathcal{H}_{3}$ inside $\bar{M}_{3}$. The cohomology class of this divisor in terms of the standard generators $\lambda, \Delta_{i}$ for $H^{2}\left(\bar{M}_{g}\right)$, up to a positive rational multiple, is given by

$$
\left[\overline{\mathcal{H}}_{3}\right]=9 \lambda-\Delta_{0}-3 \Delta_{1} \text {. }
$$

Technically in the above form we have given the relationship in the Picard group of the moduli functor and not the cohomology (Chow) ring of the moduli space: the discrepancy arises because of the presence of an orbifold structure on the entire component of the stable divisor comprising curves with elliptic tails and on the locus of hyperelliptic curves itself. Thus translating instead to the Chow group (and abusing notation by using the same symbols to denote the respective generators) we have

$$
9 \lambda-\Delta_{0}-3 \Delta_{1} \mapsto 18 \lambda-2 \Delta_{0}-3 \Delta_{1}
$$

in our examples $\Delta_{1}$ will vanish, and the re-scaling will be inconsequential. The particular advantage of working with the hyperelliptic divisor is the well-known restriction on Lefschetz pencils giving rise to spheres with image contained inside it [7]:

(4.6) Lemma (Endo) Let $X \rightarrow \mathbb{S}^{2}$ be the total space of a genus three hyperelliptic Lefschetz fibration. Then

$$
\sigma(X)=-\frac{4}{7} i+\frac{1}{7} r
$$

where $i, r$ denote the numbers of irreducible and reducible singular fibres respectively ${ }^{2}$.

There are various proofs of this result. For topologists, hyperelliptic fibrations are globally double branched covers of sphere bundles over spheres and this

\footnotetext{
${ }^{2}$ Denoting the total number of singular fibres by $s$ we arrive at the taxing formula $i+r=s$.
} 
yields an explicit signature formula. For geometers, the (nearly ample) Hodge class $\lambda$ restricts to the moduli space $\mathcal{H}_{3}$ as a certain union of boundary divisors since the open locus of hyperelliptic curves is affine, and the signature formula results from comparing to (3.4). Endo's original proof is an algebraic formulation of the last statement, analysing the signature cocycle in the second cohomology of the symplectic group under restriction to the hyperelliptic mapping class group. In any case, we have a new obstruction to holomorphic structures on genus three fibrations:

(4.7) Proposition Let $X \rightarrow \mathbb{S}^{2}$ be a genus three Lefschetz fibration with irreducible fibres. Suppose that

(1) $e(X)+1$ is not divisible by 7 ;

(2) $9 \sigma(X)+5 e(X)+40<0$.

Then the Lefschetz fibration is not isotopic to a holomorphic fibration.

Proof If $e+1$ is not divisible by seven then the integrality of the signature and Endo's formula show that the fibration is not hyperelliptic. On the other hand, the condition that $9 \sigma(X)+5 e(X)+40<0$ is exactly equivalent to the statement that $\left[\mathbb{S}_{X}^{2}\right] \cdot \mathcal{H}_{3}<0$ and so the fibration cannot yield a sphere isotopic to a rational curve.

We now hit a catch. There are rather few known genus three fibrations which admit exceptional sections, and the numerology conspires against us: for none of these does applying the relation $\mathcal{T}$ yield a negative algebraic intersection with the hyperelliptic divisor. Indeed for most we cannot apply $\mathcal{T}$ at all, since no sum of two vanishing cycles is homologically trivial, as must be the case for the curves $U, W$ in Figure 2. On the other hand, if we apply $\mathcal{T}^{-1}$ then we increase the intersection with the hyperelliptic divisor. Since the only explicitly known relations correspond to holomorphic fibrations, this would appear to be a losing strategy. Fortunately, there is a loophole. We can start with a hyperelliptic holomorphic fibration, for which the rational curve lies inside $\mathcal{H}_{3}$ and has sufficiently negative intersection with the divisor that applying $\mathcal{T}^{-1}$ does not destroy that property; but by an easy count, it will necessarily destroy the hyperellipticity. It is fairly easy to classify holomorphic hyperelliptic fibrations with no reducible fibres; to satisfy the constraints of (4.7) we are reduced to essentially a single possible example! In fact the $\mathcal{T}^{-1}$-substitution, on precisely this positive relation, had already been derived by Terry Fuller. His motivation was rather different; he wanted an unusual mapping class group word to which he could apply Kirby calculus to study covering spaces. He kindly donates the following manipulations. 
(4.8) Example Terry Fuller Let the curves $A_{i}, B_{i}, D_{2}, E_{2}$ on a genus three curve be as drawn in Figure 3 and write $a_{i}$ etc. for the positive Dehn twist about $A_{i}$. Fuller obtains the following positive relation:

$$
\left(d_{2} e_{2} b_{2} a_{2} b_{1} a_{1} a_{3} b_{2} a_{2} b_{1} b_{3} a_{3} b_{2} a_{2}\left(a_{1} b_{1} a_{2} b_{2} a_{3} b_{3}\right)^{10}\right)=1 .
$$

We begin with well-known braid relations (which do not signify any change to

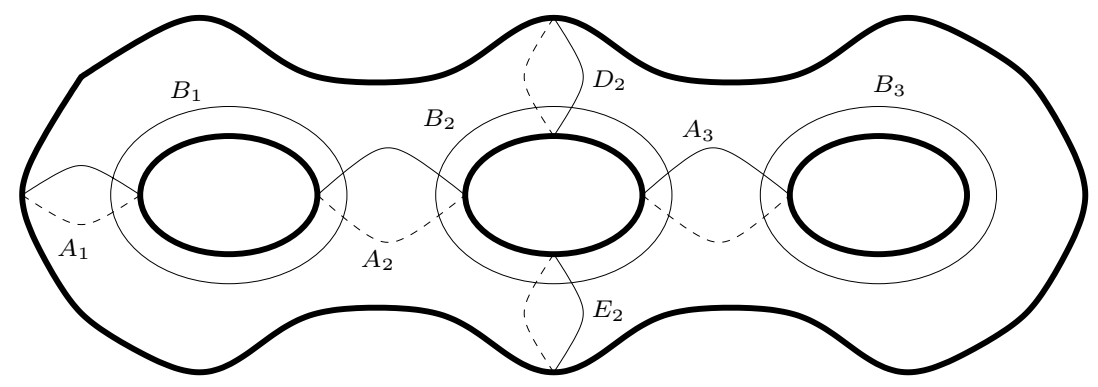

Figure 3: Supporting curves for a genus three positive relation

the four-manifold but only to its presentation as a positive relation):

$$
a_{i} b_{i} a_{i}=b_{i} a_{i} b_{i} ; \quad a_{i+1} b_{i} a_{i+1}=b_{i} a_{i+1} b_{i} .
$$

Using these freely, along with the fact that Dehn twists about disjoint curves commute, Fuller shows:

$$
\left(a_{1} b_{1} a_{2} b_{2} a_{3} b_{3}\right)^{2}=\left(a_{1} b_{1} a_{2}\right)^{2} b_{2} a_{2} a_{3} b_{2} b_{3} a_{3} .
$$

Using (4.10), and writing

$$
\left(a_{1} b_{1} a_{2} b_{2} a_{3} b_{3}\right)^{4}=\left(\left(a_{1} b_{1} a_{2} b_{2} a_{3} b_{3}\right)^{2}\right)^{2}
$$

a further sequence of braid manipulations brings you to

$$
\left(a_{1} b_{1} a_{2} b_{2} a_{3} b_{3}\right)^{4}=\left(a_{1} b_{1} a_{2}\right)^{4} b_{2} a_{2} b_{1} a_{1} a_{3} b_{2} a_{2} b_{1} b_{3} a_{3} b_{2} a_{2} .
$$

Now employ the $\mathcal{T}^{-1}$ substitution to the relation given below:

$$
\left(a_{1} b_{1} a_{2}\right)^{4}=d_{2} e_{2} ; \quad\left(a_{1} b_{1} a_{2} b_{2} a_{3} b_{3}\right)^{14}=1
$$

and combine together (4.10) and (4.11):

$$
\begin{aligned}
\left(a_{1} b_{1} a_{2} b_{2} a_{3} b_{3}\right)^{14} & =\left(a_{1} b_{1} a_{2} b_{2} a_{3} b_{3}\right)^{4}\left(a_{1} b_{1} a_{2} b_{2} a_{3} b_{3}\right)^{10} \\
& =\left(a_{1} b_{1} a_{2}\right)^{4} b_{2} a_{2} b_{1} a_{1} a_{3} b_{2} a_{2} b_{1} b_{3} a_{3} b_{2} a_{2}\left(a_{1} b_{1} a_{2} b_{2} a_{3} b_{3}\right)^{10} \\
& =d_{2} e_{2} b_{2} a_{2} b_{1} a_{1} a_{3} b_{2} a_{2} b_{1} b_{3} a_{3} b_{2} a_{2}\left(a_{1} b_{1} a_{2} b_{2} a_{3} b_{3}\right)^{10} .
\end{aligned}
$$

This gives us the relation we require. 
The relation $\left\langle\left(a_{1} \ldots b_{3}\right)^{14}=1\right\rangle$ defines the monodromy of a hyperelliptic surface obtained by taking the double branched cover of $\mathbb{F}_{2}$ over a curve in the class $7\left|s_{0}\right| \amalg\left|s_{\infty}\right|$, where $s_{0}, s_{\infty}$ denote the sections of square \pm 2 respectively. Since the section of square two lies inside the branch locus, it lifts to an exceptional section of the genus three fibration; if we blow this down we obtain a simply connected complex surface of general type. To prove that this does indeed give the genus three fibration we require, consider the curve in $\left|7 s_{0}\right|$ given locally by $z_{1}^{7}+z_{2}^{14}=0$. Deform the singularity to a union of 14 simple tangencies between distinct sheets of the curve. At each of these points the new equation is equivalent to $u^{2}+v^{7}=0$ in suitable holomorphic co-ordinates, which gives the monodromy of the $(2,7)$-torus knot (which is fibred of genus three). Then compute that the monodromy of this knot is indeed given by the product $\left(a_{1} b_{1} a_{2} b_{2} a_{3} b_{3}\right)$.

Write $W$ for the total space of the Lefschetz fibration defined by (4.8). The pencil of curves, both before and after the modification by $\mathcal{T}^{-1}$, has only irreducible fibres and hence does define a canonical symplectic structure by the theorem of Gompf mentioned in the Introduction. The number of singular fibres in the modified pencil is 74 and hence $e(W)=66$. Moreover the signature of the Horikawa surface is -48 (by Endo's formula, say) and so the modified manifold has $\sigma(W)=-42$. (This checks with the result obtained, via a computer-implemented algorithm based on Wall's non-additivity, by Ozbagci in [17]. In particular the signature is computable from the mapping class group word, even if the derivation of that word is not available!) It is now a triviality to apply (4.7) and deduce that we have indeed obtained a symplectic non-Kähler pencil. This completes the proof of (1.3).

(4.12) Remark $W$ is homeomorphic to a simply connected complex surface. To the author's knowledge, the only way to prove that it might not be Kähler is to compute instanton or Seiberg-Witten invariants. Such computations seem intractable for manifolds presented via positive relations; note that we have deduced that the Lefschetz structure is not holomorphic without needing to determine whether $W$ is diffeomorphic to a Kähler surface.

We remark for completeness that we also find large classes of non-holomorphic genus three Lefschetz fibrations on manifolds homeomorphic to complex surfaces. These examples are elementary in the sense that they do not rely on theorems from gauge theory or Donaldson's construction.

(4.13) Example A hyperelliptic genus three Lefschetz fibration $Z$ with $\delta$ singular fibres, all irreducible, has $\delta=7 r$ for some integer $r$ and topological in- 
variants $e=7 r-8, \sigma=-4 r$. Now under a fibre summation the signature and Euler characteristic transform as

$$
\sigma=\sigma_{1}+\sigma_{2} ; \quad e=e_{1}+e_{2}-2 e(F) .
$$

It follows immediately that $Z \sharp_{\Sigma_{3}} W$ will satisfy (4.7) and also the Noether inequality:

$$
\begin{gathered}
(9 \sigma+5 e+40)\left(Z \sharp_{F} W\right)=(9 \sigma+5 e+40)(W)-r \\
\left(5 c_{1}^{2}-c_{2}+36\right)\left(Z \sharp_{F} W\right)=\left(5 c_{1}^{2}-c_{2}+36\right)(W)+3 r .
\end{gathered}
$$

One can also check that for any $Z$ there is a minimal complex surface of general type homeomorphic to $Z \sharp_{F} W$ using the geographic criterion of ([4], VII (9.1)). Indeed for these manifolds it is known that there is a simply connected such complex surface [18]. By similar manipulations the reader can check the relevant numbers in the case of fibre summing $W$ with itself. Note that many simplyconnected hyperelliptic genus three fibrations are Kähler, but the holomorphic structure of the fibrations is lost on summing with $W$.

By a result of Stipsicz [25] we know that many of these manifolds are minimal. Auroux (private conversation) has noted that one can improve the first condition in (4.7) to demanding only that $e(X)+1$ be not divisible by 14 , by studying the braid factorisation for a hyperelliptic fibration instead of just the mapping class group factorisation.

(4.14) Remark Suppose we have a Lefschetz pencil which is not holomorphic, for instance by arguments as above. If the canonical symplectic form is isotopic to a Kähler form, then we can use the Riemann-Roch theorem to estimate the number of sections of the line bundle $\mathcal{L}_{C}$ with first Chern class dual to the fibre $C$ of the pencil. If any section of this line bundle had smooth zero set, then a generic pencil of sections would define a Lefschetz pencil by Bertini's theorem and general position arguments. The existence of such a pencil may well be ruled out by the topological constraints on the homology class of the sphere, which is determined by the global topology of the manifold. However, in general the line bundle $\mathcal{L}_{C}$ is ample and not necessarily very ample, and it seems impossible (even in examples) to prove directly that if there is a Kähler structure on the manifold then there would be some smooth complex curve in the linear system $|C|$. This prevents us from answering our original Question (2) in (2.1).

The situation for Question (3) is even worse: we expect the homology classes realised by our pencils to contain no smooth complex curve at all. In any complex 
surface with a homology class with an immersed but not embedded holomorphic representative, we can smooth nodes to build symplectic submanifolds which are uninterestingly distinct from complex curves.

The results of this section can be generalised to other divisors, for instance the divisor of trigonal curves in $\bar{M}_{5}$, but the author is not aware of further applications or phenomena.

\section{$5 \quad$ Weierstrass divisors and sections}

We give a final application of (2.7) with a result on sections of fibrations that one can prove in a similar vein. This adds to the now substantial body of knowledge on genus two fibrations [24]. Recall the symplectic isotopy conjecture due to Siebert and Tian [19] states that every connected symplectic submanifold of a relatively minimal sphere bundle over a sphere is isotopic to a complex submanifold ${ }^{3}$.

(5.1) Proposition Let $f: X \rightarrow \mathbb{S}^{2}$ be a genus two Lefschetz fibration and $s: \mathbb{S}^{2} \rightarrow X$ a distinguished section of $f$. Suppose the fibration has $\delta_{0}$ irreducible singular fibres, $\delta_{1}$ reducible ones and $\delta_{0}+2 \delta_{1}=10 m$ for $m \in \mathbb{Z}$. Suppose also that the symplectic isotopy conjecture is valid. Then

(1) $3|s \cdot s| \geq m+\delta_{1}$ or

(2) $4|s \cdot s|=m+\delta_{1}$.

Proof Inside $\bar{M}_{2}^{1}$, the moduli space of stable curves with a single marked point - equivalently the universal curve $\overline{\mathcal{C}}_{2}$ over $\bar{M}_{2}$ - there is a divisor $\mathcal{W}$ comprising the closure of pairs $(C, p)$ where $p$ is one of the Weierstrass points of $C$. In the notation of [11], the cohomology of $\bar{M}_{2}^{1}$ is generated by elements $\omega_{R D}, \lambda, \delta_{0}, \delta_{1}$, where $\omega_{R D}$ is the relative dualising sheaf and $\lambda$ the pullback of the Hodge bundle under $\bar{M}_{2}^{1} \rightarrow \bar{M}_{2}$. The adjunction formula allows us to identify the value of the class $\omega_{R D}$ on our family with the negative of the self-intersection of the distinguished section:

$$
\left\langle\omega_{R D},[B]\right\rangle=-s \cdot s .
$$

\footnotetext{
${ }^{3}$ In lectures in June 2001, Siebert and Tian have announced a partial resolution of this conjecture which is probably strong enough to show that genus two Lefschetz fibrations without reducible fibres are holomorphic. This would suffice for this Proposition.
} 
With respect to this basis, we have an identity in the Picard group of the moduli functor

$$
[\mathcal{W}]=3 \omega_{R D}-\lambda-\delta_{1}
$$

Let $f: X \rightarrow \mathbb{P}^{1}$ be a genus two Lefschetz fibration with a distinguished section $s: \mathbb{P}^{1} \rightarrow X$. Recall from [24] that we can write $X$ as a double cover of a rational ruled manifold Rat $_{X}$ over a locus which is smooth away from finitely many infinitely close triple points (their number given by the number of reducible fibres in the Lefschetz fibration). Moreover, on any fibre the branch covering map can be identified with the hyperelliptic involution on the fibre after choosing metrics: that is, the ramification locus is precisely the closure of the union of the Weierstrass points in each fibre.

Via the fixed section $s$ we induce a map $\mathbb{S}^{2} \rightarrow \bar{M}_{2}^{1}$. If the map has image inside the divisor $\mathcal{W}$ then the section lies inside the locus of Weierstrass points and gives rise to a branch locus which is disconnected. Siebert and Tian [19] analyse the branch locus for any hyperelliptic fibration branched over a rational ruled manifold and show that it has at most two components, and if it is not connected then one of the components is a sphere section for the natural fibration of the ruled surface over $\mathbb{S}^{2}$. In this case the self-intersection of the sphere is given by $-k$ where the base can be identified with $\mathbb{P}(\mathcal{O} \oplus \mathcal{O}(k))$. (Warning: Note here that $k$ is even and $-k$ is the self-intersection of the sphere as a submanifold of the base; the natural lift of the sphere to the ramification locus has square $-k / 2$.)

On any Lefschetz fibration, there is an operation which removes a reducible fibre and replaces it by a sequence of $(4 h+2) 2 h$ irreducible fibres, where $h$ is the genus of one component of the reducible fibre. Thus for a genus two fibration we can remove a reducible fibre and replace it by twelve irreducible ones. This operation can be localised downstairs in the branched covers and corresponds to resolving or deforming an infinitely close triple point singularity. It follows from this description that if there is a section disjoint from the singularity, one can trade the two local models without changing the section or its square. Thus we have an operation on genus two fibrations which has the numeric effect

$$
\delta_{1} \mapsto \delta_{1}-1 ; \delta_{0} \mapsto \delta_{0}+12 ; m \mapsto m+1 ;|s \cdot s| \mapsto|s \cdot s| .
$$

If we trade all the reducible fibres for irreducible ones, we arrive at a fibration which is (modulo the symplectic isotopy conjecture for surfaces of appropriate bidegree) necessarily Kähler.

In this case, the rational curve it defines in $\bar{M}_{2}^{1}$ is either contained in $\mathcal{W}$ or has locally positive intersections with it. Since $3|s \cdot s|-m-\delta_{1}$ is unchanged by the 
removal of the reducible fibres, we obtain the two cases of the proposition. For suppose this value is negative. Then the rational curve lies inside $\mathcal{W}$ and defines a hyperelliptic fibration with disconnected branch locus. Then by Siebert-Tian the self-intersection of the section component of the fibration is precisely $-k / 2$ where the base of the hyperelliptic double cover is $\mathbb{P}(\mathcal{O} \oplus \mathcal{O}(k))$. Moreover by a result of [24], we can relate this value $k$ to the number of critical fibres of the fibration: precisely, $|k|=m$ where there are $10 m$ critical fibres.

Since we assume the symplectic isotopy conjecture, a genus two Lefschetz fibration with only irreducible fibres is holomorphic, and such objects were classified by Chakiris and independently by the author [24]. The only irreducible fibrations are listed in the following proposition (5.5). Using the classification, and the explicit constructions of [24], one can check that if the ramification locus contains a section component, then its square is related to the number of critical fibres $10 m$ by the formula $|s \cdot s|=m / 4$. Since after trading away the reducible fibres this number $m$ is given by $m+\delta_{1}$ for the original fibration, we find in the end that $4|s \cdot s|=m+\delta_{1}$ as claimed.

One consequence of this result is a strong restriction on which genus two Lefschetz fibrations can admit sections of square $(-1)$. This corollary can be obtained by other methods, which may be of interest in themselves. In Figure 3 we depict a genus three curve: if we cut this along the cycles $D_{2}$ and $E_{2}$ and glue the boundary curves of the left component, we obtain a genus two curve with distinguished cycles $A_{1}, B_{1}, A_{2}, B_{2}$ and $D_{2}=E_{2}=A_{3}$. Again we will use lower case letters to denote the associated Dehn twist diffeomorphisms, now of the genus two curve. The idea of this second proof is that it is easier to restrict the topology of Lefschetz pencils than Lefschetz fibrations. Recall that we showed in $(2.9)$ that the Poincaré dual of the fibre $[C] \in H_{2}(X ; \mathbb{Z})$ of a pencil was not always represented by a symplectic form adapted to the fibration. If there were such a form, and if the four-manifold satisfied the constraint $b_{+}>1$, then by Taubes' results on the canonical class we would deduce that $K_{X} \cdot[C] \geq 0$ with equality if and only if $K_{X}=0$. This helpful property persists:

(5.4) Proposition Let $X$ be a smooth four-manifold with a Lefschetz pencil of curves each representing a homology class $[C]$. Suppose that $b_{+}(X)>1$. Then the canonical deformation equivalence class of symplectic forms on $X$ defined by the pencil gives an almost complex structure such that $K_{X}$ satisfies

$$
K_{X} \cdot[C] \geq 0 ; \quad K_{X} \cdot[C]=0 \Rightarrow K_{X}=0 .
$$


Proof The canonical class for the associated Lefschetz fibration represents $\pi^{*} K_{X}+\sum E_{i}$ in homology, where the $E_{i}$ are the exceptional curves and $\pi: X^{\prime} \rightarrow$ $X$ the blow-down map. By adjunction, we know that $K_{X^{\prime}} \cdot\left[\pi^{*} C\right]=\operatorname{deg}\left(K_{C}\right)=$ $2 g-2$. Moreover we know that for any almost complex structure $J$, there is an immersed holomorphic representative for $K_{X}$ and that pseudoholomorphic curves have locally positive intersections. Choosing $J$ so that the exceptional spheres are indeed pseudoholomorphic, it follows that $[C] \cdot[C] \leq 2 g-2$ with equality iff $K_{X^{\prime}}=\sum E_{i}$. But this implies the proposition.

Armed with this we can establish some control on the homotopy types of manifolds with genus two pencils ${ }^{4}$.

(5.5) Theorem Let a symplectic four-manifold $X$ admit a Lefschetz pencil of genus two curves. Then there are only finitely many possibilities for the numbers $n, s$ of irreducible and reducible critical fibres, or equivalently for the pair $\left(c_{1}^{2}(X), c_{2}(X)\right)$. In particular $e(X)<40$. If $s=0$ then the fibration determined by the pencil on $X$ is homeomorphic to one of the simply connected complex surfaces associated to the three monodromy words:

(1) $\left(a_{1} b_{1} a_{2} b_{2} a_{3} a_{3} b_{2} a_{2} b_{1} a_{1}\right)^{2}=1$;

(2) $\left(a_{1} b_{1} a_{2} b_{2} a_{3}\right)^{6}=1$;

(3) $\left(a_{1} b_{1} a_{2} b_{2}\right)^{10}=1$.

Proof Be given $X$ and write $[C]$ for the homology class defined by a fibre of the pencil. Assume $b_{+}(X)>1$; then our proposition (5.4) tells us that $2 g-2=2=K_{X} \cdot[C]+[C]^{2}$ with both of the terms in the last expression non-negative, and the first zero only if $K_{X}=0$. This gives a small number of possibilities: either $K_{X}=0$ and $\omega^{2}=2$, or $K_{X} \cdot \omega=1$ and $\omega^{2}=1$. Suppose first that $K_{X}=0$; then $c_{1}^{2}=2 e+3 \sigma=0$. The Euler characteristic $e$ and signature $\sigma$ are determined by the numbers of critical fibres. Let there be $n$ non-separating critical fibres and $s$ separating ones. Then

$$
e=n+s-6 ; \quad \sigma=\frac{3}{5} n-\frac{1}{5} s+2
$$

where we have used the formulae for the associated fibration of curves from [24] and the fact that the pencil has two base-points. It follows from these formulae that

$$
n+7 s=30
$$

\footnotetext{
${ }^{4} \mathrm{~A}$ stronger version of this result follows from combining the isotopy conjecture and the first result of the section.
} 
whilst (for any genus two fibration, since $\left(\Gamma_{2}\right)_{\mathrm{ab}}=\mathbb{Z}_{10}$ ) also $n+2 s$ is divisible by 10 . This gives two possibilities: $n=30, s=0$ and $n=16, s=2$. In the first case, we know the fibration is simply-connected [19] and (since $K_{X}=0$ ) it is minimal. An easy computation then shows that it is homeomorphic to the $K 3$ surface blown up twice, which is described by the second word listed in the statement of the proposition. In fact a symplectic four-manifold with $\pi_{1}=0, c_{1}=0$ is necessarily homeomorphic to the $K 3$ surface by a result of Morgan and Szabo [16].

Suppose instead that $\omega^{2}=1$. Let $X^{\prime}=X \sharp \overline{\mathbb{C P}}^{2}$ be the total space of the Lefschetz fibration. By Taubes, we have an immersed holomorphic curve representing $K_{X^{\prime}}$ and containing the exceptional section. If the symplectic representative for $K_{X^{\prime}}$ is smooth, then it meets each genus two fibre two times; since it contains the exceptional section, it must comprise two disjoint sections. But by adjunction this gives a smooth (symplectic) genus zero representative for $K_{X}$, which is therefore an exceptional sphere. Thus $X$ is the blow-up of a simply connected manifold with $K_{X}=0$, and by the result of Morgan-Szabo referred to above we see that the given pencil of curves is just the blow-up of the usual genus two pencil on $K 3$ at one of its two base-points. In particular, the associated fibration is the one obtained above.

The only other possibility is that the symplectic subvariety provided by Taubes is in fact composed of several components. This subvariety meets every genus two fibre with which it shares no component locally positively and with algebraic intersection number two. Hence the curve must comprise the exceptional section counted to multiplicity two, and a number $r$ of fibres of the fibration. Suppose we write $K_{X^{\prime}}=2 E+r F$; then $K_{X^{\prime}}^{2}=4 r-4$ and so $K_{X}^{2}=4 r-3$, blowing down again. However, this is also given by $g-1$ where $g$ is the genus of the smooth symplectic representative for $K_{X}$ obtained by Taubes for a generic complex structure. (Here we define genus via a sum over components if necessary). By construction, one symplectic representative for $K_{X}$ is given by a number $r$ of the genus two curves of the pencil smoothed at the base-point. The result is that

$$
2 r=4 r-2
$$

and hence $r=1$. But then this determines $c_{1}^{2}\left(X^{\prime}\right)=0$ and this is enough to fix the number of critical fibres of the fibration, using the usual formulae for $e, \sigma$. This gives the finiteness we require. If we also know $s=0$ and the fibration is simply connected, and since the intersection form must be odd as $\left(K_{X}\right)^{2}=1$, we have determined $X$ to homeomorphism. It must be a surface of general type, with $K_{X}=[\omega]$ represented by a genus two curve of square one. Such is 
described by the third word in the list of monodromies given in the statement of the theorem.

The last remaining case is where Taubes does not apply, that is $b_{+}(X)=1$. In this case

$$
\sigma=1-b_{-}=\frac{3}{5} n-\frac{1}{5} s+\omega^{2}
$$

and

$$
e=n+s-4-\omega^{2}=3-2 b_{1}+b_{-} .
$$

Moreover we still know that $K_{X} \cdot \omega+\omega^{2}=2$ and $b_{1}=0$ or $b_{1}=2$, since $b_{1}, b_{+}$have opposite parity on any almost complex four-manifold and for any Lefschetz fibration $b_{1}$ of the total space is strictly smaller than $b_{1}$ of the fibre. For a fibration with $s=0$ we know we have simply connected total space, so $b_{1}=0$ and some easy manipulations give $\sigma+e=4$ and then $n=20$. To homeomorphism this gives the manifold given by the first monodromy word on the list above. The other cases, with $s \neq 0$ and either $b_{1}$ are also easily listed: in all cases $n \leq 20$ and hence only finitely many pairs $(n, s)$ arise.

This establishes the last of the theorems given in the opening section of the paper. Although determining a symplectic manifold only to homeomorphism is a very weak statement, the limited geography of manifolds with genus two pencils is striking in its own right. It would be very interesting to understand if there is any analogue of this at higher genera.

\section{References}

[1] E Arabello, M Cornalba, The Picard groups of the moduli spaces of curves, Topology, 26 (1987) 153-171

[2] D Auroux, Symplectic four-manifolds as branched coverings over $\mathbb{C P}^{2}$, Invent. Math. 139 (2000) 551-602

[3] D Auroux, L Katzarkov, The degree doubling formula for braid monodromies and Lefschetz pencils, Preprint (2000)

[4] W Barth, C Peters, A Van de Ven, Compact complex surfaces, Springer (1984)

[5] S K Donaldson, I Smith, Lefschetz pencils and the canonical class for symplectic 4-manifolds, Preprint (2000) arxiv:math.SG/0012067

[6] S K Donaldson, Lefschetz pencils on symplectic manifolds, J. Diff. Geom. 53 (1999) 205-236 
[7] H Endo, Meyer's signature cocyle and hyperelliptic fibrations, Math. Ann. 316 (2000) 237-257

[8] R Fintushel, R Stern, Counterexamples to a symplectic analogue of a theorem of Arakelov and Parsin, Preprint (1999)

[9] R Gompf, The topology of symplectic manifolds, Turkish J. Math. 25 (2001) 43-59

[10] J Harer, The second homology group of the mapping class group of an orientable surface, Invent. Math. 72 (1983) 221-239

[11] J Harris, I Morrison, Moduli of curves, Springer (1998)

[12] A Liu, Some new applications of the general wall-crossing formula, Math. Res. Lett. 3 (1996) 569-585

[13] F Luo, A presentation of the mapping class groups, Math. Res. Lett. 4 (1997) 735-739

[14] Y Matsumoto, Lefschetz fibrations of genus two - a topological approach, The 37th Taniguchi Symposium on topology and Teichmüller spaces (S Kojima et al, eds.) World Scientific (1996)

[15] D McDuff, D Salamon, A survey of symplectic 4 -manifolds with $b_{+}=1$, Turkish J. Math. 20 (1996) 47-61

[16] J Morgan, Z Szabo, Homotopy K3 surfaces and mod 2 Seiberg-Witten invariants, Math. Res. Lett. 4 (1997) 17-21

[17] B Ozbagci, Signatures of Lefschetz fibrations, Pacific J. Math. (to appear)

[18] U Persson, Chern invariants of surfaces of general type, Comp. Math. 43 (1981) 3-58

[19] B Siebert, G Tian, On hyperelliptic $C^{\infty}$-Lefschetz fibrations of fourmanifolds, Commun. Contemp. Math. 1 (1999) 466-488

[20] I Smith, Gauge theory and symplectic linear systems, In preparation

[21] I Smith, Geometric monodromy and the hyperbolic disc, Quarterly J. Math. (Oxford) 52 (2001) 217-228

[22] I Smith, Serre-Taubes duality for pseudoholomorphic curves, Preprint (2001)

[23] I Smith, Symplectic geometry of Lefschetz fibrations, Ph.D. thesis, Oxford University (1998)

[24] I Smith, Lefschetz fibrations and the Hodge bundle, Geometry and Topology 3 (1999) 211-233

[25] A Stipsicz, On the number of vanishing cycles in Lefschetz fibrations, Math. Res. Lett. 6 (1999) 449-456

[26] A Stipsicz, On the indecomposability of certain Lefschetz fibrations, Proc. Amer. Math. Soc. 129 (2001) 1499-1502

[27] C H Taubes, The Seiberg-Witten and the Gromov invariants, Math. Res. Letters, 2 (1995) 221-238 